\title{
Prenatal glucocorticoid exposure in rats: programming effects on stress reactivity and cognition in adult offspring
}

\author{
Yan Zeng ${ }^{1}$, Nichola M. Brydges ${ }^{2}$, Emma R. Wood ${ }^{3}$, Amanda J. Drake ${ }^{1 *}$, and Jeremy Hall ${ }^{2,4,5 *}$ \\ ${ }^{1}$ University/BHF Centre for Cardiovascular Science, QMRI, University of Edinburgh, Edinburgh, UK, ${ }^{2}$ Neuroscience and Mental Health Research \\ Institute, Cardiff University School of Medicine, Cardiff, UK, ${ }^{3}$ Centre for Cognitive and Neural Systems and Centre for Cognitive Aging and Cognitive \\ Epidemiology, School of Biomedical Sciences, University of Edinburgh, Edinburgh, UK, ${ }^{4}$ Division for Psychiatry, Centre for Clinical Brain Science, \\ University of Edinburgh, Edinburgh, UK, and ${ }^{5}$ MRC Centre for Neuropsychiatric Genetics and Genomics, Cardiff University School of Medicine, \\ Cardiff, UK
}

\begin{abstract}
Human epidemiological studies have provided compelling evidence that prenatal exposure to stress is associated with significantly increased risks of developing psychiatric disorders in adulthood. Exposure to excessive maternal glucocorticoids may underlie this fetal programming effect. In the current study, we assessed how prenatal dexamethasone administration during the last week of gestation affects stress reactivity and cognition in adult offspring. Stress reactivity was assessed by evaluating anxiety-like behavior on an elevated plus maze and in an open field. In addition, to characterize the long-term cognitive outcomes of prenatal exposure to glucocorticoids, animals were assessed on two cognitive tasks, a spatial reference memory task with reversal learning and a delayed matching to position (DMTP) task. Our results suggest that prenatal exposure to dexamethasone had no observable effect on anxiety-like behavior, but affected cognition in the adult offspring. Prenatally dexamethasone-exposed animals showed a transient deficit in the spatial reference memory task and a trend to faster acquisition during the reversal-learning phase. Furthermore, prenatally dexamethasone-treated animals also showed faster learning of new platform positions in the DMTP task. These results suggest that fetal overexposure to glucocorticoids programs a phenotype characterized by cognitive flexibility and adaptability to frequent changes in environmental circumstances. This can be viewed as an attempt to increase the fitness of survival in a potentially hazardous postnatal environment, as predicted by intrauterine adversity. Collectively, our data suggest that prenatal exposure to dexamethasone in rats could be used as an animal model for studying some cognitive components of related psychiatric disorders.
\end{abstract}

\section{Keywords}

Anxiety, water maze, flexibility, elevated plus maze, open field

\section{History}

Received 17 November 2014

Revised 30 April 2015

Accepted 2 May 2015

Published online 6 July 2015

\section{Introduction}

Early life environmental events, such as maternal stress, infection and/or immune activation and nutritional deficiencies can have programming effects on the developing fetus. These factors can alter the physiology and functioning of developing organs, thus increasing the vulnerability of the offspring to develop adulthood diseases (Barker, 1990). Large-scale epidemiological studies from diverse human populations suggest that an aversive prenatal environment is associated with an increased risk of developing psychiatric

This is an Open Access article distributed under the terms of the Creative Commons Attribution License (http://creativecommons.org/ Licenses/by/4.0/), which permits unrestricted use, distribution, and reproduction in any medium, provided the original work is properly cited.

*Dr. Drake and Professor Hall are joint senior authors on this paper.

Correspondence: Dr Nichola M. Brydges, Neuroscience and Mental Health Research Institute, Cardiff University School of Medicine, Hadyn Ellis Building, Cardiff, UK. Tel: +44 (0)2920688342. E-mail: brydgesn@cardiff.ac.uk disorders later in life, including autism spectrum disorders (ASD), schizophrenia and attentional deficit/hyperactivity disorder (ADHD) (Kinney, 2000; Kinney et al., 2008; Linnet et al., 2003; Rodriguez \& Bohlin, 2005). The timing of the adverse exposure is thought to be critical in determining programming effects. Current evidence suggests that stressors in the middle of gestation or before birth are critical to the development of ASD (Beversdorf et al., 2005); exposures during the first (Susser \& Lin, 1992; Van Os \& Selten, 1998) or second trimester (Huttunen \& Niskanen, 1978; Van Os \& Selten, 1998) are linked to schizophrenia, while exposures only during the second trimester associate with the development of ADHD (Van den Bergh \& Marcoen, 2004).

A number of human and animal studies also provide robust evidence that prenatal stress can lead to persistent neuropsychological abnormalities in the offspring (Koehl et al., 2001; Talge et al., 2007). In rodents, prenatal stress programs hypersensitivity to stress, including heightened hypothalamicpituitary-adrenal (HPA) axis activity (Vallee et al., 1997; Wilson et al., 2013) and increased fear and anxiety-like behavior (Takahashi et al., 1992; Vallee et al., 1997; Wakshlak 
\& Weinstock, 1990; Welberg et al., 2001). Prenatal stress also affects cognition as demonstrated by impaired spatial learning and memory (Brabham et al., 2000; Lemaire et al., 2000; Markham et al., 2010; Son et al., 2006; Yang et al., 2006), working memory (Gue et al., 2004; Markham et al., 2010) and cued and contextual fear conditioning and extinction (Green et al., 2011; Markham et al., 2010; Wilson et al., 2013).

In our current study, we treated pregnant rats with dexamethasone, a synthetic glucocorticoid that crosses the placental glucocorticoid barrier, during the last week of gestation. Rodent brain development at this time resembles that in the second trimester of human gestation (Clancy et al., 2007), the time-point most strongly linked to adulthood psychiatric disorders. Previous studies in this model have shown that this manipulation programs behavioral inhibition in adult rat offspring (Welberg et al., 2001), however, fewer studies have investigated any cognitive consequences. We aimed to investigate the impact of prenatal glucocorticoid overexposure on (i) adult stress reactivity by evaluating anxiety-like behavior, using the elevated plus maze and open field tests and (ii) cognition, using a spatial reference memory task with reversal learning, followed by a delayed matching to position (DMTP) task. These experiments enabled us to investigate the effects of prenatal glucocorticoid exposure on offspring stress reactivity and a range of cognitive phenotypes of relevance to neuropsychiatric disorders.

\section{Materials and methods}

\section{Animals}

Subjects were adult Wistar rats, bred from 30 adult pairs (Charles River, UK) at the University of Edinburgh. Animals had ad libitum access to food (standard rat chow, RM1, Special Services Diet, Lillico, Surrey, UK) and water. Rats were maintained under conditions of controlled temperature (between 19 and $21^{\circ} \mathrm{C}$ ) and light/dark cycle (lights on $12 \mathrm{~h}$ per day from 07:00 to 19:00). All experiments were performed in accordance with the UK Animals Act (Scientific Procedures) 1986 and were conducted strictly according to local ethics guidelines to minimize animal suffering and the number of animals to be used.

Female rats were housed individually and timed mating was conducted. Following vaginal plugging (denoted as E1) pregnant dams were randomly assigned to dexamethasone (Dex) or vehicle (Con) treatment. From E15 to E21 inclusive, dams were given a daily subcutaneous injection of either dexamethasone $(100 \mu \mathrm{g} / \mathrm{kg}$ body weight, dissolved in $4 \%$ ethanol, $0.9 \%$ saline) or an equivalent volume of vehicle ( $4 \%$ ethanol, $0.9 \%$ saline) $(0.5 \mathrm{ml} / \mathrm{kg})$.

At birth (postnatal day 1), the offspring (Dex or Con) were weighed, sexed and litters were culled back to eight per group, leaving five males and three females per litter where possible. Litters were then left undisturbed until weaning at 3 weeks of age, whereupon females were culled and male pups were housed in same groups and left undisturbed until adulthood. For each behavioral test, we used one or two rats per litter for each group, and in the case of using two rats from the same litter the effect of litter was accounted for in the statistical analysis.

\section{Plasma corticosterone levels}

Animals were assessed for baseline plasma corticosterone (CORT) levels in adulthood (aged 139.61 \pm 1.33 days, $n=9-14$ per group). Blood samples were collected from the tail vein at two time points, 07.00 and $19.00 \mathrm{~h}$. Rats were taken from their home cage and directly sampled with minimal stress. All blood samples were collected by capillary blood collection tube (Microvette ${ }^{\circledR}$ 300) and centrifuged for $5 \mathrm{~min}$ at $13,000 \mathrm{rpm}$ at room temperature. The plasma was then transferred and stored at $-80^{\circ} \mathrm{C}$ until analysis. Plasma CORT concentrations were analyzed using enzyme-linked immunosorbent assay (Al-Dujaili et al., 2009).

\section{Elevated plus maze}

Animals were aged $72.0 \pm 3.95$ days at test $(n=10-11$ per group). The elevated plus maze was a cross-shaped platform positioned $80 \mathrm{~cm}$ above the floor with two open arms and two closed arms $(70 \mathrm{~cm} \times 12 \mathrm{~cm} \times 17 \mathrm{~cm})$ on opposing sides of a central area $(10 \mathrm{~cm} \times 10 \mathrm{~cm})$. At the start of the test a rat was put in the central area facing one of the open arms and allowed to explore the maze freely for $5 \mathrm{~min}$, during which its behavior was recorded by a video camera mounted above the maze. Following each session the apparatus was wiped clean with $70 \%$ ethanol to remove any olfactory cues. An experimenter blind to treatment groups handled the animals and analyzed the video recordings. The following parameters were considered: number of open arm entries, the amount of time spent on the open arms and total number of arm entries.

\section{Open field}

Animals were aged $70.75 \pm 4.46$ days at test $(n=12$ per group). The open field apparatus was a black square box $(90 \mathrm{~cm} \times 90 \mathrm{~cm} \times 60 \mathrm{~cm}$ high). The field was divided into nine equal segments. At the start of the test, a rat was placed at the edge of the box, in the middle of one of the surrounding walls and allowed to explore the field freely for $5 \mathrm{~min}$, during which its behavior was recorded by a video camera mounted above the maze. Starting position was counterbalanced between groups. The field was cleaned by $70 \%$ ethanol to remove any possible olfactory cues between each animal. An experimenter blind to treatment groups handled the animals and analyzed the video recordings. The parameters of interest were determined as: the total number of grid crossings, the amount of time spent in the central square and number of central square entries. One week after the open field test, the animals were tested on the DMTP task.

\section{Morris water maze}

Spatial learning and memory tests took place in an open-field water maze (Morris, 1984). This was a water tank of $2 \mathrm{~m}$ in diameter and $0.5 \mathrm{~m}$ in depth, filled with water made opaque by mixing it with $300 \mathrm{ml}$ of liquid latex (temperature $\left.25 \pm 1^{\circ} \mathrm{C}\right)$. An escape platform $(12 \mathrm{~cm}$ in diameter) was submerged $1.5 \mathrm{~cm}$ below the water surface. The maze was surrounded by a collection of prominent two- and threedimensional visual cues. The animal's behavior was monitored using a video tracking system via a camera fixed above the centre of the maze, and was analyzed using water maze 
software (Tracker-P. Spooner, Edinburgh, UK). For each trial, the latency to reach the platform, path length and swimming speed were measured. Four points of equal spacing along the circumference of the pool were signified as starting points: North (N), East (E), South (S) and West (W). Likewise, the swimming pool was conceptually divided into four quadrants accordingly, NE, SE, SW and NW.

\section{Spatial reference memory with reversal learning}

Animals were assessed for spatial reference memory and reversal-learning task at age $100.38 \pm 6.39$ days at the start of testing ( $n=12$ per group). This experiment consisted of 5 days of initial training and 5 days of reversal learning. For each rat, the platform location remained constant (either NE or SW quadrant, $60 \mathrm{~cm}$ from the wall, counterbalanced between groups) throughout the first 5 days of training, and was moved to the opposite quadrant (this time SW or NE) for the following 5 days of reversal learning. On each day, rats were given five trials in a row, with an inter-trial interval (ITI) of approximately $15 \mathrm{~s}$. At the start of each trial the rat was carefully lowered into the water, facing one of the four starting points. Starting position was counterbalanced between groups. The sequence of the starting positions was randomized, with at least one start at N, E, S and W on each day, avoiding repeats for two consecutive trials. The first trial of each day was run as a probe trial. Probe trials used the "Atlantis" platform (Spooner et al., 1994), which was pushed down at first to render it unavailable and set to rise at $60 \mathrm{~s}$ by using a computercontrolled electromagnet, and the animal was given a further $30 \mathrm{~s}$ to find it. The percentage of time spent near the initial training platform location (radius $20.0 \mathrm{~cm}$ ) during the first $60 \mathrm{~s}$ was analyzed. The following trials on each day (trials 2-5) were standard trials, in which rats were allowed $120 \mathrm{~s}$ to find the platform, before being ushered toward it. Once the animal reached the platform, it was allowed to remain on the platform for $30 \mathrm{~s}$ before being taken off and moved on to the next trial.

\section{Delayed matching to position}

Animals were assessed for DMTP task at age $90.92 \pm 12.11$ days at the start of testing ( $n=12$ per group). The DMTP task comprised 7 days of initial training followed by three blocks of delay tests, each of these blocks continued for 5 days. The platform location was different on each day, but remained the same for all the trials of that day. The daily platform location was randomly selected from one of the 28 possible locations situated in an inner $(1 \mathrm{~m}$ in diameter) and outer $(1.5 \mathrm{~m}$ in diameter) ring of the swimming pool. Each rat received four trials on a given day, the starting position for each trial was considered in relevance to the location of the platform, designated as far right (FR), far left (FL), near right (NR), near left (NL), and randomized for the four trials of that day. The sequences of the daily platform locations and relative starting position were carefully designed to counterbalance between and within groups to avoid any clear recognizable pattern.

\section{DMTP - training phase}

During the training phase all rats were given four consecutive trials per day for 7 days. The platform was placed according to the pre-designed location, and submerged $1.5 \mathrm{~cm}$ below the water surface before the trials began. On each trial the rat was allowed $120 \mathrm{~s}$ to find the platform, before being guided toward it. Once the rat climbed on the platform, it had $30 \mathrm{~s}$ to remain on the platform. An experimenter would then remove the rat and allow a $15 \mathrm{~s}$ ITI before the next trial.

\section{DMTP - delay phase}

During the delay phase, the training protocol was the same as before, except that a delay time of $15 \mathrm{~s}, 20 \mathrm{~min}$ or $2 \mathrm{~h}$ was introduced between the first and the second trial, during which time animals were dried and returned to their home cage. Each animal experienced all three delays, in a counterbalanced fashion. Rats were initially assigned to one of the three delays, and remained on that delay for 5 days. After 2 days' break, rats moved onto another delay, then another 2 days' break followed by 5 days of the final delay. Probe tests used the same "Atlantis" platform as detailed above, and were carried out on the second trial on the last day of each block. The percentage of time spent near the most recently trained platform location (radius $20.0 \mathrm{~cm}$ ) during the first $60 \mathrm{~s}$ of the probe trial was analyzed, to allow for assessment of working memory.

\section{Data analysis}

Data were analyzed using generalized linear models (JMP statistical software, SAS Institute, Cary, NC), followed by Tukey's honest significant difference post hoc test, where appropriate. All data were checked for homogeneity of variance and normality of distribution. All statistical models investigated the main effects of treatment. Animal identity (nested within litter and group) and litter (nested within group) were fitted as random variables to account for repeated measurements and the use of more than one animal per litter.

For the spatial reference memory and reversal-learning task, models were used to investigate the effects of Group, Trial, Day and all two-, three-way interactions of these terms on latency to platform, path length and average swimming speed for all the standard trials (trials 2-5). For probe trials (trial 1) only, models were used to investigate the effects of Group, Day and two-way interactions of these terms on percentage of time near the initial training platform.

For the DMTP task, data were analyzed separately. For the training phase, models were used to investigate the effect of Group, Trial, Day and all two-, three-way interactions of these terms on latency to platform, path length and average swimming speed for all the trials from the training phase. For the delay phase, models were employed to investigate the effect of Group, Delay, Trial and all two-, three-way interactions of these terms on latency to platform, path length and average swimming speed for all the trials from the first 4 days of each block (excluding the last day of each block). For probe trials (the second trial on the last day of each block), models were used to investigate the effect of Group, Delay and two-way interactions of these terms on percentage of time near the most recently trained platform locations, divided by the total amount of time spent near the other seven related platform locations, sitting at the vertices of an octagon. Data are presented as mean value per group \pm SEM. 


\section{Results}

\section{Birth weight}

Prenatal dexamethasone treatment significantly reduced birth weight $\left(F_{1,28.45}=12.85, p=0.0012\right)$. Female pups were significantly smaller than their male counterparts at birth $\left(F_{1,415.3}=39.00, \quad p<0.0001\right)$. There was no interaction between Group and Sex.

\section{Plasma CORT levels}

Figure 1 shows that the typical diurnal pattern of plasma CORT concentrations was seen in both experimental groups. No significant differences were found between the two experimental groups in either the morning $\left(F_{1,5.55}=0.12\right.$, $p=0.738)$, or evening CORT levels $\left(F_{1,8.34}=0.47\right.$, $p=0.512)$.

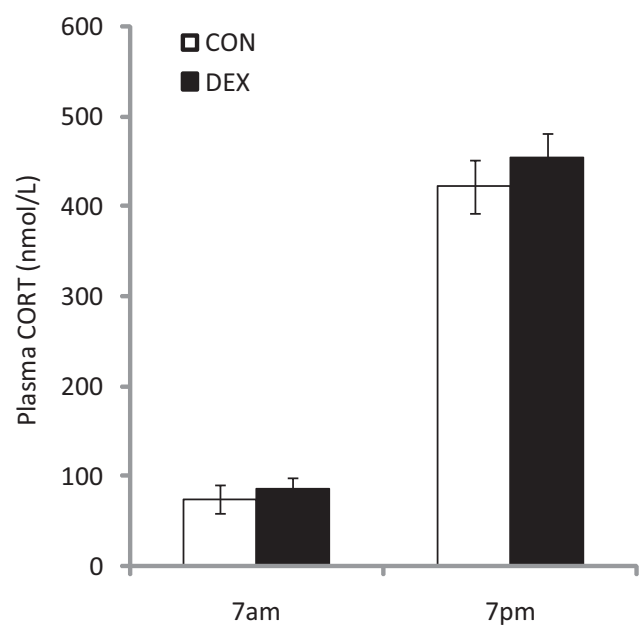

Figure 1. Effects of prenatal exposure to dexamethasone on adult offspring plasma corticosterone (CORT) levels. There were no significant differences between the two experimental groups in CORT levels measured at 7 am and $7 \mathrm{pm}$. Data are presented as mean \pm SEM. $n=9-14$ per group.

Figure 2. Performance on an elevated plusmaze. There were no significant differences between the two experimental groups on number of open arm entries (A); the amount of time spent on open arms (B) or number of total arm entries (C). Data are presented as mean \pm SEM. $n=10-11$ per group.

\section{Elevated plus maze}

Figure 2 illustrates performance on the elevated plus maze. Groups did not differ in their number of entries to the open arms $\left(F_{1,8.21}=0.61, p=0.458\right)$, the amount of time spent on the open arms $\left(F_{1,9.81}=0.51, p=0.492\right)$ or total number of arm entries $\left(F_{1,19}=2.41, p=0.137\right)$.

\section{Open field}

Figure 3 illustrates performance during the open field test ( $n=12$ per group). Prenatal dexamethasone exposure had no significant effect on total grid crossings $\left(F_{1,8.02}=4.00\right.$, $p=0.080)$, amount of time spent in the central square $\left(\mathrm{F}_{1,9,95}=1.25, p=0.290\right)$, or number of central square entries $\left(F_{1,7.93}=2.10, p=0.186\right)$.

\section{Spatial reference memory with reversal learning Latency to platform}

Figure 4 demonstrates performance on the spatial reference memory and reversal-learning task. There was a significant effect of Day on latency to find the platform $\left(F_{9,917}=62.27\right.$, $p<0.0001)$. No significant effect of Group was found $\left(F_{1,3.68}=1.60, p=0.281\right)$, however, there was a significant interaction between Group and Day $\left(F_{9,917}=2.40, p=0.011\right)$. Post hoc analysis showed that Dex animals were significantly slower than controls in finding the platform on the first day of initial training $(p<0.05)$. Both groups improved their performance on the following days and by day 4 all had reached a stable level of behavioral performance. In addition, on the first day of the reversal phase, there was a trend toward a difference between groups such that control animals took a longer time to find the new platform location, although this did not reach statistical significance (Figure 4A).

\section{Performance on probe trials}

Figure 4(B) shows the percentage of time spent in a zone near the initial training platform (radius $20.0 \mathrm{~cm}$ ) on probe trials.
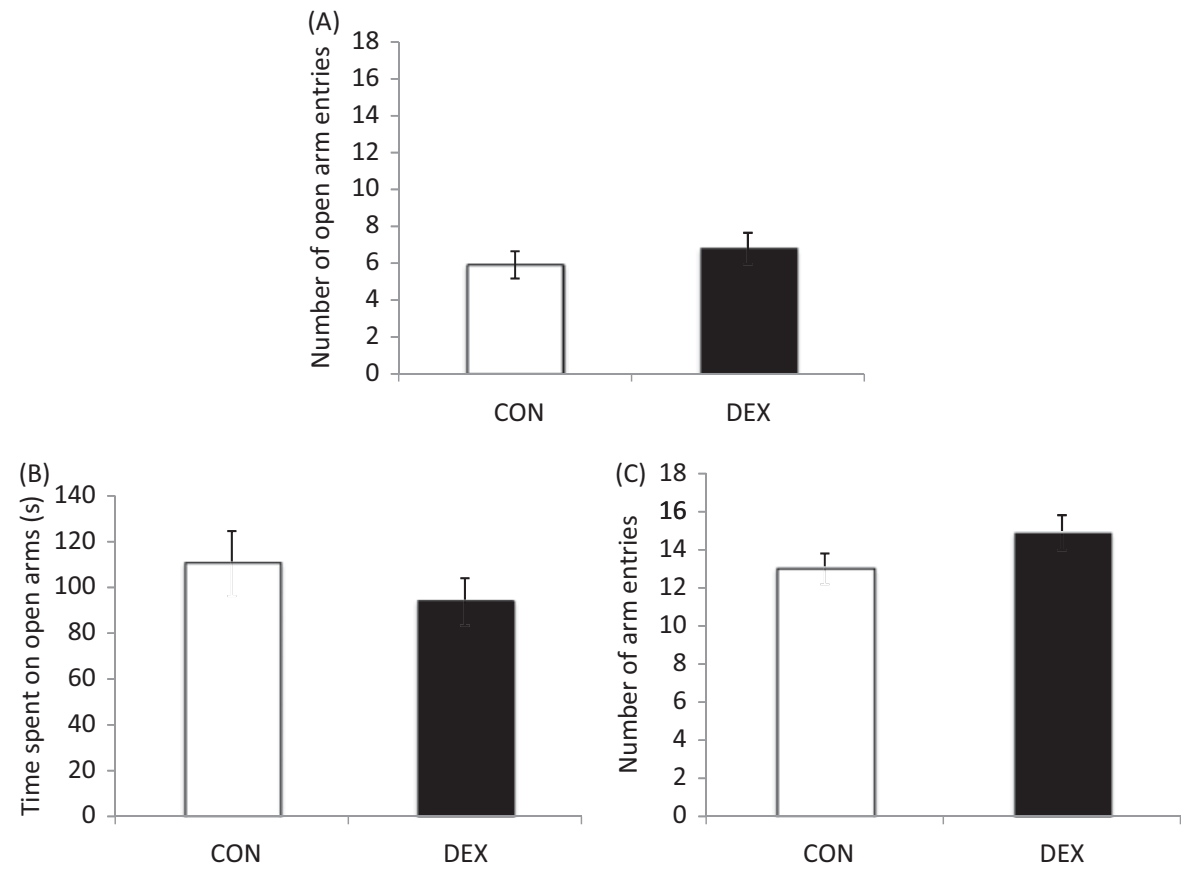
There was a significant effect of Day $\left(F_{9,189}=11.35\right.$, $p<0.0001$ ), such that all animals spent increasingly more time around the training platform as days progressed, reaching a stable level by day 5 . No significant difference was found between groups in probe trial performance $\left(F_{1,4.722}=0.03\right.$, $p=0.863)$, nor was there any interaction between Group and Day $\left(F_{9,189}=0.72, p=0.690\right)$.

\section{Locomotor activity}

There were no significant differences between the groups in average swimming speed during the whole experiment period $\left(F_{1,4.50}=0.71, F=0.443\right)$.

\section{Delayed matching to position}

DMTP - latency to platform in training phase

Figure 5(A) illustrates the latency to platform during the training phase. Statistical analysis revealed a significant effect of Day $\left(F_{6,594}=31.86, \quad p<0.0001\right)$, such that animals improved their performance as days progressed, reaching a steady level by day 4 . There was also a significant effect of Trial within each day $\left(F_{3,594}=31.35, p<0.0001\right)$, as animals took significantly longer time to find the platform on the first trial compared to the other three. Moreover, there was a significant effect of Group $\left(F_{1,7.69}=8.16, p=0.022\right)$, with Dex animals taking significantly less time than controls to find the platform. There was no significant interaction between Group and Day $\left(F_{6,594}=0.68, p=0.666\right)$. An identical pattern of performance was seen when path length was used as the dependent variable (data not shown).

\section{DMTP - latency to platform in delay phase}

There was no significant effect of Delay $\left(F_{2,1034}=0.36\right.$, $p=0.70)$, or any interaction between Group and Delay $\left(F_{2,1034}=1.95, p=0.142\right)$. However, similar to the findings from the training phase, there was a significant effect of Group, with Dex animals taking significantly less time to find the platform than controls $\left(F_{1,9.592}=5.58, p=0.041\right)$ (Figure 5B).

\section{DMTP - performance on probe trials}

Probe trial data were analyzed as the probability of swimming near the training platform compared to the other seven related locations, sitting at the vertices of an octagon. There was no significant effect of Group $\left(F_{1,8.332}=1.65, p=0.23\right)$, no significant effect of Delay $\left(F_{2,44}=0.59, p=0.558\right)$ and no interaction between Group and Delay $\left(F_{2,44}=0.48\right.$, $p=0.621)$. The average probability of swimming around the training platform location was $0.245 \pm 0.140$ for all animals, above the chance level, which was 0.125 for our analysis (Figure 5C).

\section{DMTP - locomotor activity}

There were no significant differences between the groups in average swimming speed $\left(F_{1,8.01}=0.68, F=0.432\right)$ during the whole experiment period.

\section{Discussion}

In the present study, we found that prenatal exposure to dexamethasone during the last week of gestation in rats decreased birth weight. However, there was no treatment effect on stress reactivity in a range of physiological and behavioral measures. Nevertheless, prenatally dexamethasone-exposed rats demonstrated altered cognition in water maze tests. This was represented by: (1) a transient deficit on the first day of the spatial reference memory task and (2) significantly faster locating of new platform positions in the DMTP task in the dexamethasone group. Collectively, these data suggest that prenatal dexamethasone-exposure affected cognition in the adult offspring, producing a phenotype
Figure 3. Performance in a 5-min open field test. Prenatal dexamethasone exposure had no significant effects on total grid crossings (A); time spent in the central square (B) or number of entries to the centre (C). Data are presented as mean \pm SEM. $n=12$ per group.
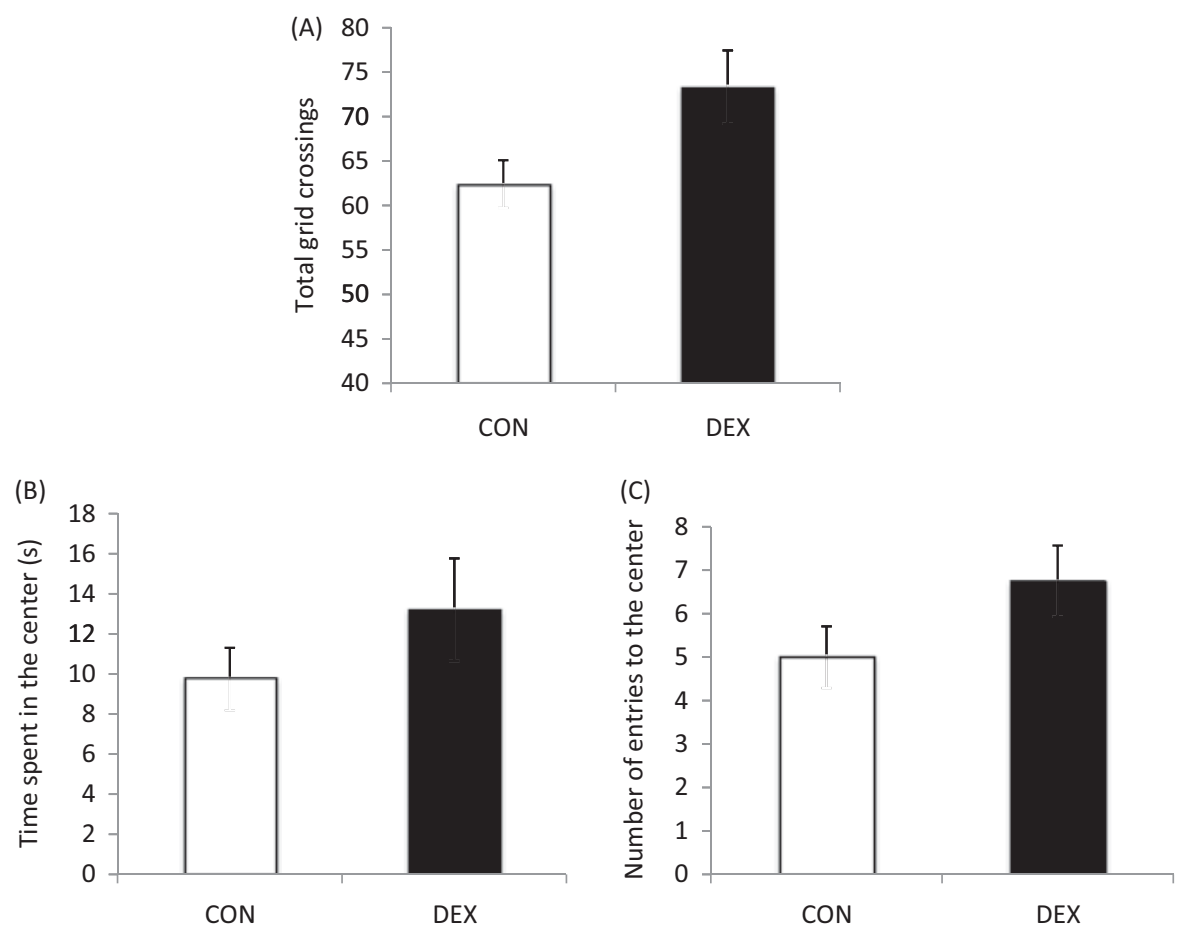

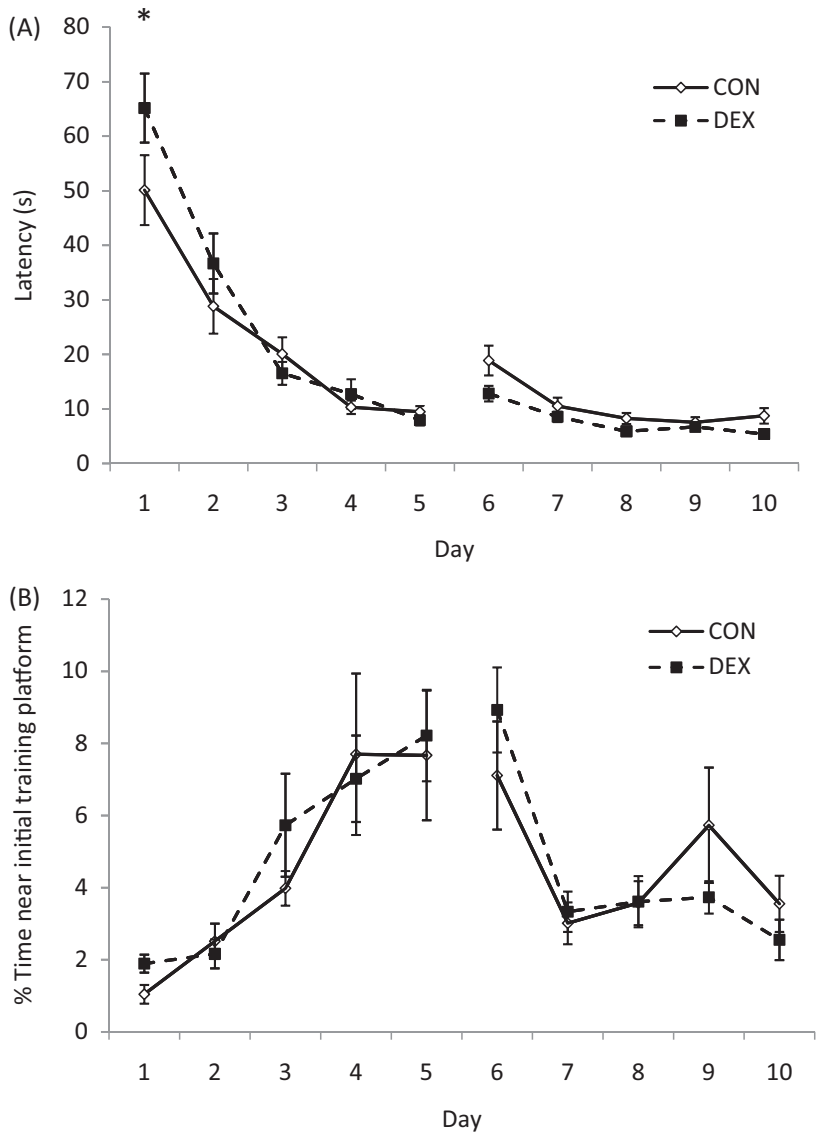

Figure 4. Performance on a spatial reference memory with reversallearning task in the Morris water maze. (A) Animals exposed to prenatal dexamethasone showed a significant interaction between group and day in latency to platform $(p=0.011)$. Data represent the average of all standard trials (trials 2-5) presented on each day. (B) Both groups of animals showed a similar preference for the training platform location when memory was probed $24 \mathrm{~h}$ after the last trial (first trial of each day). Data are presented as mean \pm SEM. $n=12$ per group. ${ }^{*} p<0.05$ versus control.

characterized by responsive flexibility and adaptability to changing situations.

Our data confirmed that dexamethasone treatment during the last week of gestation in rats reduced birth weight in offspring, in line with previous findings (Welberg et al., 2001). Recent evidence suggests that low birth weight is predictive of neuropsychiatric diseases in adult life, including autism (Pinto-Martin et al., 2011), schizophrenia (Abel et al., 2010) and ADHD (Breslau et al., 1996). Thus, this animal model may provide a laboratory tool to study the mechanisms underlie this link. There was no effect of prenatal glucocorticoid exposure on baseline CORT levels in our study, consistent with some previous results (Wilson et al., 2013). However, this does not exclude an effect on HPA axis activity, since one of the best-characterized deficits in HPA axis regulation induced by prenatal stress is the negative feedback control of stress-induced hormone responses (Vallee et al., 1997; Wilson et al., 2013). Additional assessment of HPA axis responses to stress, measurement of circulating ACTH and the temporal patterns of activation and termination of HPA hormone secretion after repeated exposure to stress (Fride et al., 1986) would provide further insight into longterm effects in this model.
We further investigated between-group differences in behavioral reactivity to stress using the elevated plus-maze and open field, which revealed no effect of prenatal dexamethasone exposure on adult anxiety-like behavior. Although similar negative findings have been reported by others (Wilson et al., 2013), our results contrast with a previous study using the same animal model (Welberg et al., 2001). The reasons for this are unclear but may include differences in experimental conditions, such as the use of different experimental apparatus and the setting (these experiments were performed in a different animal facility). To clarify the discrepancy, we sought to replicate our results with two other cohorts of rats in the same and different animal facilities. Despite variations in rearing environment and experimental settings, our results from the three cohorts were consistent.

Importantly, our results from the two water maze spatial learning and memory tests demonstrated altered cognition in prenatally dexamethasone-exposed rats. The spatial reference memory task has been validated as a robust measure of hippocampus-dependent spatial navigation and reference memory (D'Hooge \& De Deyn, 2001). The reversal-learning phase requires the animal to withhold a formerly learned response and shift to a new stimulus-reward contingency, and the neural substrate underlying the reversal has been ascribed to the prefrontal cortex (Lacroix et al., 2002). From the spatial reference memory and reversal-learning task, we found that prenatal dexamethasone exposure induced a transient impairment in spatial short-term memory seen on the first day of initial training. However, prenatally exposed rats were able to improve their performance and caught up with controls on the second day, indicating that their spatial reference memory was not impaired. This was further supported by performance on probe trials, in which all animals showed similar preferences to swim in the vicinity of the training platform. Taken together, our experiment suggests that prenatal dexamethasone treatment had no sustained effect on hippocampusrelated spatial reference memory, but induced a transient deficit in processing short-term spatial memory in the adult offspring. In addition, when presented with a spatial reversal, there was a trend toward a group difference, with Dex animals locating the new platform position faster than controls. These novel results are potentially consistent with a deficit in prefrontal cortex function (Debruin et al., 1994).

These results led us to conduct a second experiment, which investigated in more detail the effects of repeated learning of novel spatial information. To do this we performed a DMTP task, which draws on many of the cognitive functions ascribed to prefrontal cortex, including working memory and behavioral flexibility that represent key functions of the prefrontal cortex (Kesner, 2000). The "delay" part of the DMTP task allows assessment of working memory, including short-term retention of visuo-spatial information and the executive functions of coordinating and manipulating this information to plan for subsequent responses (Baddeley, 1992). The "matching" component of the DMTP task requires frequent shifts in response to daily changes in stimulus-related spatial information, allowing for the assessment of behavioral flexibility. An efficient performance on the DMTP task depends critically on the integrity of reciprocal interactions 
Figure 5. Performance on a delayed matching to position task (DMTP) in the Morris water maze. There was a significant effect of group in both the training phase (A), and delay phase (B), so that rats exposed to prenatal dexamethasone had shorter latencies to reach the platform. Data are averaged over delays and days during the delay phase. All animals showed equivalent performance on probe trials after any of a $15 \mathrm{~s}, 20 \mathrm{~min}$, or $2 \mathrm{~h}$ delay (C). Data are presented as mean \pm SEM. $n=12$ per group. $* p<0.05$ versus control.
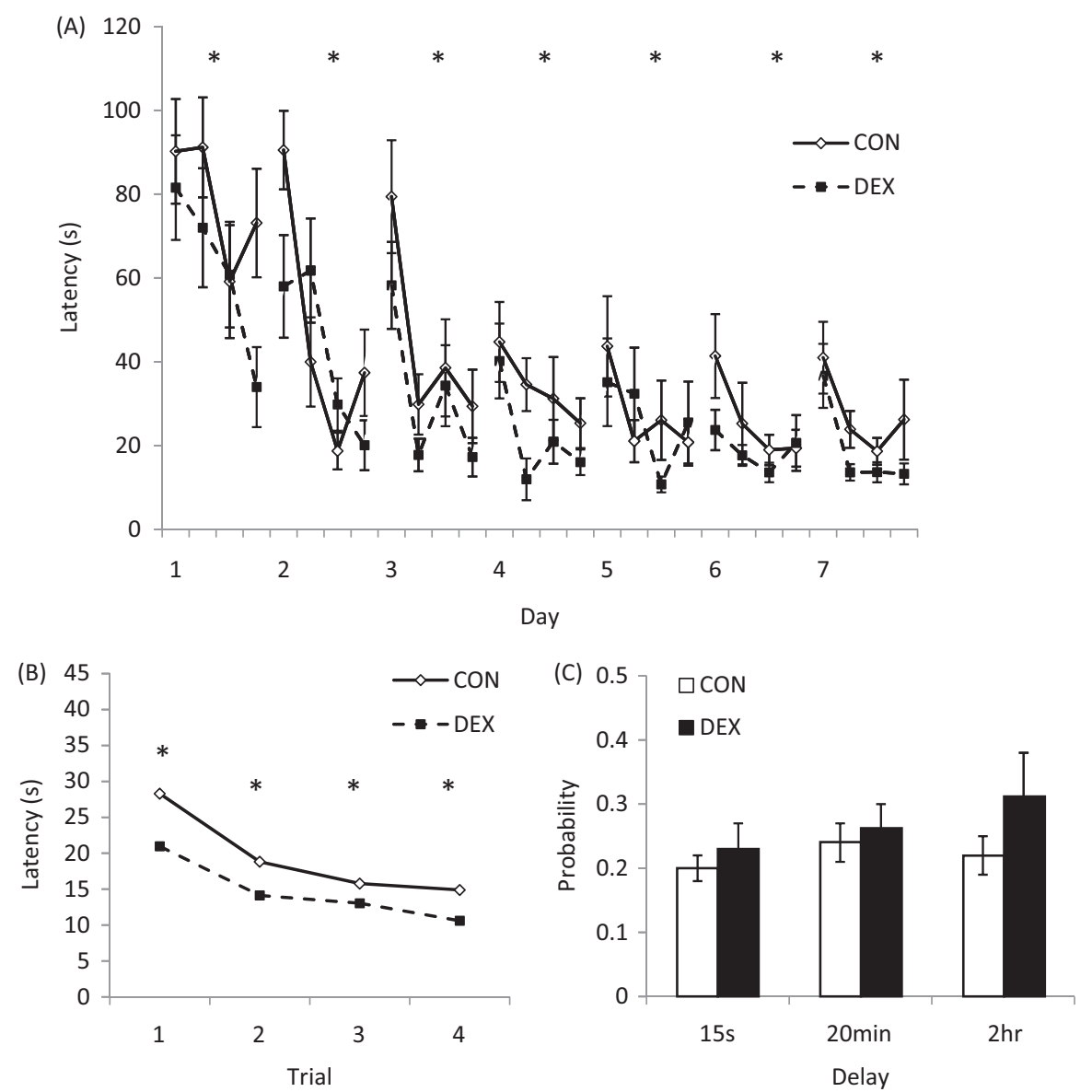

between the hippocampus and prefrontal cortex (Floresco et al., 1997). Our data from the DMTP task revealed a significant between-group difference, with Dex animals taking significantly less time than controls to find the platform, potentially consistent with greater cognitive flexibility, or a lesser adherence to a pre-existing cognitive set. The fact that prenatally stressed animals demonstrated enhanced performance on the DMTP task (which has daily changes of platform positions) suggests that they were faster in responding to tasks that require frequent shifting of responses to changes in stimulus-reward contingencies. The reversal-learning task required only one shift at the reversal stage, whereas in the DMTP task the rule was changing every day, and therefore demanded much more flexibility in shifting their responses from one to another. Thus, the results from our current study reflect different abilities in responding to changes in environmental circumstances between the two groups. One potential explanation of these findings was that Dex-exposed animals showed less perseveration to the previous platform position. However, within the current dataset we were not able to find direct evidence for difference in perseveration, and additional experiment would be required to investigate this issue further.

Thus, our present study suggests that prenatal dexamethasone exposure affects cognition in adult offspring, characterized by a phenotype of cognitive flexibility and adaptability to frequent changes in environmental circumstances. Similar features can be seen in patients afflicted by psychiatric disorders such as ADHD (Barkley, 1997), one risk factor for which is prenatal stress (Rodriguez \& Bohlin, 2005; Van den Bergh \& Marcoen, 2004). Such a cognitive outcome induced by prenatal glucocorticoid exposure could be viewed as adaptive, in that individuals who experience an aversive intrauterine environment are programmed to "expect" an unfavorable postnatal environment; this adaptive advantage would become evident if the later environment turned out to be as challenging as predicted. This is consistent with the recent "'match-mismatch" hypothesis advanced by Schmidt and others, which suggest that early life programming effects are not necessarily deleterious. Thus, the costs and benefits of a programmed trait need to be interpreted in the context of the individual and the current environment (Nederhof \& Schmidt, 2012). There is some evidence that prenatal stress alters maternal care, which might influence and further contribute to the alterations in the offspring (Francis et al., 1999; Kuo et al., 2014). Although in this experiment we were unable to dissect any contribution of prenatal and postnatal maternal effects on the programmed phenotypes in offspring, previous studies including in this model suggest that the programming effects induced by prenatal treatment are independent of maternal postnatal behavior (Holloway et al., 2013; Nyirenda et al., 2001).

In conclusion, the results from our current study suggest that prenatal exposure to glucocorticoids had no observable programming effects on stress reactivity in adult offspring, but resulted in long-lasting alterations in cognition. Prenatally dexamethasone-exposed rats showed a transient deficit in spatial short-term memory, but their spatial reference memory 
and working memory were not affected. In addition, dexamethasone-programmed rats showed faster responses during the reversal learning and the DMTP task, indicating cognitive flexibility and responsive adaptability. These novel results suggest that prenatal glucocorticoid exposure has programming effects on prefrontal cortex-related behavioral flexibility and executive functions. The phenomenological similarities between this animal model and clinical features of some neuropsychiatric disorders support the validity of using this animal model for studying risk for these conditions.

\section{Acknowledgements}

The authors thank Dr. Emad Al-Dujaili for assistance with the CORT assay.

\section{Declaration of interest}

The authors declare no financial, consulting or personal conflicts of interest in relation to the work presented. The experiments described in this manuscript were supported in part by grants from the China Scholarship Council, The Waterloo Foundation, the RS MacDonald Trust and the Medical Research Council (MR/L010305/1). J.H. and A.J.D. were supported by Scottish Senior Clinical Fellowships.

\section{References}

Abel KM, Wicks S, Susser ES, Dalman C, Pedersen MG, Mortensen PB, Webb RT. (2010). Birth weight, schizophrenia, and adult mental disorder: is risk confined to the smallest babies? Arch Gen Psychiatry 67:923-30.

Al-Dujaili EA, Mullins LJ, Bailey MA, Andrew R, Kenyon CJ. (2009). Physiological and pathophysiological applications of sensitive elisa methods for urinary deoxycorticosterone and corticosterone in rodents. Steroids 74:938-44.

Baddeley A. (1992). Working memory. Science 255:556-9.

Barker DJ. (1990). The fetal and infant origins of adult disease. BMJ 301:1111.

Barkley RA. (1997). Behavioral inhibition, sustained attention, and executive functions: constructing a unifying theory of adhd. Psychol Bull 121:65-94.

Beversdorf DQ, Manning SE, Hillier A, Anderson SL, Nordgren RE, Walters SE, Nagaraja HN, et al. (2005). Timing of prenatal stressors and autism. J Autism Dev Disorders 35:471-8.

Brabham T, Phelka A, Zimmer C, Nash A, Lopez JF, Vazquez DM. (2000). Effects of prenatal dexamethasone on spatial learning and response to stress is influenced by maternal factors. Am J Physiol Regul Integr Comp Physiol 279:R1899-909.

Breslau N, Brown GG, Deldotto JE, Kumar S, Ezhuthachan S, Andreski P, Hufnagle KG. (1996). Psychiatric sequelae of low birth weight at 6 years of age. J Abnorm Child Psychol 24:385-400.

Clancy B, Finlay BL, Darlington RB, Anand KJ. (2007). Extrapolating brain development from experimental species to humans. Neurotoxicology 28:931-7.

Debruin JPC, Sanchezsanted F, Heinsbroek RPW, Donker A, Postmes P. (1994). A behavioral-analysis of rats with damage to the medial prefrontal cortex using the morris water maze - evidence for behavioral flexibility, but not for impaired spatial navigation. Brain Res 652:323-33.

D’hooge R, De Deyn PP. (2001). Applications of the morris water maze in the study of learning and memory. Brain Res Brain Res Rev 36: $60-90$.

Floresco SB, Seamans JK, Phillips AG. (1997). Selective roles for hippocampal, prefrontal cortical, and ventral striatal circuits in radialarm maze tasks with or without a delay. J Neurosci 17:1880-90.

Francis D, Diorio J, Liu D, Meaney MJ. (1999). Nongenomic transmission across generations of maternal behavior and stress responses in the rat. Sci 286:1155-8.
Fride E, Dan Y, Feldon J, Halevy G, Weinstock M. (1986). Effects of prenatal stress on vulnerability to stress in prepubertal and adult rats. Physiol Behav 37:681-7.

Green MK, Rani CS, Joshi A, Soto-Pina AE, Martinez PA, Frazer A, Strong R, Morilak DA. (2011). Prenatal stress induces long term stress vulnerability, compromising stress response systems in the brain and impairing extinction of conditioned fear after adult stress. Neuroscience 192:438-51.

Gue M, Bravard A, Meunier J, Veyrier R, Gaillet S, Recasens M, Maurice T. (2004). Sex differences in learning deficits induced by prenatal stress in juvenile rats. Behav Brain Res 150:149-57.

Holloway T, Moreno JL, Umali A, Rayannavar V, Hodes GE, Russo SJ, Gonzalez-Maeso J. (2013). Prenatal stress induces schizophrenia-like alterations of serotonin $2 \mathrm{a}$ and metabotropic glutamate 2 receptors in the adult offspring: role of maternal immune system. J Neurosci 33: $1088-98$

Huttunen MO, Niskanen P. (1978). Prenatal loss of father and psychiatric disorders. Arch Gen Psychiatry 35:429-31.

Kesner RP. (2000). Subregional analysis of mnemonic functions of the prefrontal cortex in the rat. Psychobiology 28:219-28.

Kinney DK. (2000). Prenatal stress and risk for schizophrenia. Int J Mental Health 29:62-72.

Kinney DK, Munir KM, Crowley DJ, Miller AM. (2008). Prenatal stress and risk for autism. Neurosci Biobehav Rev 32:1519-32.

Koehl M, Lemaire V, Vallee M, Abrous N, Piazza PV, Mayo W, Maccari S, Le Moal M. (2001). Long term neurodevelopmental and behavioral effects of perinatal life events in rats. Neurotox Res 3: $65-83$.

Kuo HC, Guo MM, Liu SF, Chen CC, Sheen JM, Yu HR, Tiao MM, et al. (2014). Cross-fostering increases th $1 /$ th 2 expression in a prenatal dexamethasone exposure rat model. PLoS One 9:e115554.

Lacroix L, White I, Feldon J. (2002). Effect of excitotoxic lesions of rat medial prefrontal cortex on spatial memory. Behav Brain Res 133: $69-81$.

Lemaire V, Koehl M, Le Moal M, Abrous DN. (2000). Prenatal stress produces learning deficits associated with an inhibition of neurogenesis in the hippocampus. Proc Natl Acad Sci USA 97:11032-7.

Linnet KM, Dalsgaard S, Obel C, Wisborg K, Henriksen TB, Rodriguez A, Kotimaa A, et al. (2003). Maternal lifestyle factors in pregnancy risk of attention deficit hyperactivity disorder and associated behaviors: review of the current evidence. Am J Psychiatry 160:1028-40.

Markham JA, Taylor AR, Taylor SB, Bell DB, Koenig JI. (2010). Characterization of the cognitive impairments induced by prenatal exposure to stress in the rat. Front Behav Neurosci 4:173. doi: 10.3389/fnbeh.2010.00173.

Morris R. (1984). Developments of a water-maze procedure for studying spatial learning in the rat. J Neurosci Methods 11:47-60.

Nederhof E, Schmidt MV. (2012). Mismatch or cumulative stress: toward an integrated hypothesis of programming effects. Physiol Behav 106: 691-700.

Nyirenda MJ, Welberg LA, Seckl JR. (2001). Programming hyperglycaemia in the rat through prenatal exposure to glucocorticoids-fetal effect or maternal influence? J Endocrinol 170:653-60.

Pinto-Martin JA, Levy SE, Feldman JF, Lorenz JM, Paneth N, Whitaker AH. (2011). Prevalence of autism spectrum disorder in adolescents born weighing $<2000$ grams. Pediatrics 128:883-91.

Rodriguez A, Bohlin G. (2005). Are maternal smoking and stress during pregnancy related to ADHD symptoms in children? J Child Psychol Psychiatry 46:246-54.

Son GH, Geum D, Chung S, Kim EJ, Jo JH, Kim CM, Lee KH, et al. (2006). Maternal stress produces learning deficits associated with impairment of NMDA receptor-mediated synaptic plasticity. J Neurosci 26:3309-18.

Spooner RI, Thomson A, Hall J, Morris RG, Salter SH. (1994). The atlantis platform: a new design and further developments of buresova's on-demand platform for the water maze. Learn Mem 1:203-11.

Susser ES, Lin SP. (1992). Schizophrenia after prenatal exposure to the dutch hunger winter of 1944-1945. Arch Gen Psychiatry 49: 983-8.

Takahashi LK, Turner JG, Kalin NH. (1992). Prenatal stress alters brain catecholaminergic activity and potentiates stress-induced behavior in adult-rats. Brain Res 574:131-7.

Talge NM, Neal C, Glover V, Early Stress, Translational Research and Prevention Science Network: Fetal and Neonatal Experience on Child 
and Adolescent Mental Health. (2007). Antenatal maternal stress and long-term effects on child neurodevelopment: how and why? J Child Psychol Psychiatry 48:245-61.

Vallee M, Mayo W, Dellu F, Le Moal M, Simon H, Maccari S. (1997). Prenatal stress induces high anxiety and postnatal handling induces low anxiety in adult offspring: correlation with stress-induced corticosterone secretion. J Neurosci 17:2626-36.

Van den Bergh BR, Marcoen A. (2004). High antenatal maternal anxiety is related to adhd symptoms, externalizing problems, and anxiety in 8- and 9-year-olds. Child Dev 75:1085-97.

Van Os J, Selten JP. (1998). Prenatal exposure to maternal stress and subsequent schizophrenia - the may 1940 invasion of the netherlands. Br J Psychiatry 172:324-6.
Wakshlak A, Weinstock M. (1990). Neonatal handling reverses behavioral abnormalities induced in rats by prenatal stress. Physiol Behav 48:289-92.

Welberg LA, Seckl JR, Holmes MC. (2001). Prenatal glucocorticoid programming of brain corticosteroid receptors and corticotrophinreleasing hormone: possible implications for behaviour. Neuroscience 104:71-9.

Wilson CA, Vazdarjanova A, Terry Jr AV. (2013). Exposure to variable prenatal stress in rats: effects on anxiety-related behaviors, innate and contextual fear, and fear extinction. Behav Brain Res 238:279-88.

Yang J, Han H, Cao J, Li L, Xu L. (2006). Prenatal stress modifies hippocampal synaptic plasticity and spatial learning in young rat offspring. Hippocampus 16:431-6. 